\section{APRIL fait le printemps des plasmocytes}

Maka Burjanadze, Eddy Roosnek, Jakob Passweg, Bertrand Huard
M. Burjanadze, B. Huard : Division d'Hématologie, Hôpitaux Universitaires et Département de Patho-Immunologie, Centre Médical Universitaire, 1 , rue Michel Servet, 1211 Genève, Suisse. bertrand.huard@medecine.unige.ch maka.burjanadze@medecine.unige.ch દ. Roosnek, J. Passweg: Division d'Hématologie, Hôpitaux Universitaires, Centre Médical Universitaire, 1211 Genève, Suisse.
$>$ Les plasmocytes, cellules sécrétrices de nos anticorps, sont indispensables à notre protection contre les pathogènes. Il est connu que la survie de ces cellules est très dépendante de leur environnement, mais les molécules impliquées restent mal définies. Des études récentes ont révélé le rôle primordial d'APRIL (a proliferation inducing ligand), une molécule appartenant à la superfamille du tumor necrosis factor (TNF), dans la survie des plasmocytes. Ainsi, des niches riches en APRIL se forment dans la zone sous-épithéliale des muqueuses lors d'une infection, ce qui permet l'accumulation et la persistance des plasmocytes. La formation de ces niches se faisant dans un contexte inflammatoire, la survie d'un plasmocyte dans les muqueuses est donc liée à la présence d'un pathogène et ne peut être que de courte durée. Au contraire, dans la moelle osseuse, la production d'APRIL est constitutive, expliquant au moins en partie pourquoi le plasmocyte peut résider dans ce tissu pendant une longue période.

\section{Complexité biologique} de la superfamille du TNF : la sous-famille d'APRIL et BAFF

On compte à l'heure actuelle 19 ligands de la superfamille du tumor necrosis factor (TNF), mais 28 membres dans la superfamille des récepteurs du TNF (TNF-R). Cette discordance des nombres de ligands et de récepteurs s'explique par la polyvalence de certains ligands et l'existence de trois TNF- $R$ encore orphelins (TNFRSF-19 ou TROY; TNFRSF-19 like ou receptor expressed in lymphoid tissues, RELT; TNFRSF-21 ou death receptor 6 , DR6). Compte tenu de l'ensemble des données déjà obtenues grâce au séquençage de l'ADN humain au cours de ces dix dernières années, les recherches futures n'identifieront plus de nouveaux membres partageant les domaines classiques TNF ou TNF-R caractéristiques de ces superfamilles. Il y a donc fort à parier que si ces trois récepteurs orphelins possèdent un ligand, sa structure sera très différente de l'enchaînement de feuillets $\beta$, qui caractérise les domaines d'homologie au TNF. Malgré l'identification de toutes ces paires récepteurs/ ligands, beaucoup reste à faire pour comprendre la biologie de toutes ces molécules.
Les superfamilles TNF/TNF-R assurent des fonctions pléiotropes, qui peuvent aller de l'induction de la mort cellulaire à l'organogenèse. Une telle diversité de fonctions permet d'apprécier la complexité biologique de ces superfamilles, qui se décline en sous-familles incluant des membres aux fonctions similaires. APRIL ou TNFSF-13, fait partie d'une sous-famille qui inclue son cousin germain the $B$-cell activation factor from the TNF family (BAFF, TNFSF-13B). Les domaines d'homologie au TNF d'APRIL et de BAFF partagent $50 \%$ d'homologie, alors que les autres membres de la superfamille ne montrent au mieux que $16 \%$ d'homologie avec ces deux molécules. Dans ces mêmes domaines, on trouve aussi un pont disulfure intramoléculaire liant deux feuillets $\beta$, unique à BAFF et APRIL. En plus de la similitude de leur séquence protéique, BAFF et APRIL partagent une organisation génomique proche avec un intron dans la séquence nucléique codant pour leur domaine d'homologie au TNF et un exon court en amont codant pour un site de clivage par des protéases (de type furine). L'exon codant le site de clivage et le pont disulfure sont également présents dans deux autres membres de la superfamille, TWEAK (TNF-like weak

Figure 1. Phylogénie et fonction de la sous-famille APRIL. L'arbre phylogénétique de la sous-famille APRIL a été construit d'après une analyse neigbhor joining. *BALM n'a été retrouvé que chez les poissons tel que l'épinoche, la truite arcen-ciel, le poisson ballon à point noir, et le fugu. Les fonctions de ces différents gènes sont indiquées. TWEAK: TNF-like weak inducer of apoptosis ; EDA : ectodysplasin; BALM: BAFF-APRIL-like molecule; BAFF: B-cell activation factor from the TNF family. 
inducer of apoptosis, TNFSF 12) et EDA (ectodysplasin), qui sont par conséquent intégrés à la même sous-famille. Si l'on regarde les fonctions des membres de cette sous-famille, on remarque une sous-division possible. EDA a une fonction dans la morphogenèse de certains organes, APRIL et BAFF ont une fonction dans l'immunité humorale, et TWEAK, quant à lui, est multifonctionnel. Ces divergences de fonction corrèlent avec les divergences de séquence (Figure 1). De manière surprenante, on décrit chez les poissons un autre membre de cette sous-famille, appelé BAFFAPRIL-like molecule (BALM), mais qui semble unique aux poissons, car l'orthologue humain n'a pas été identifié [1]. II est donc vraisemblable que ce gène s'est individualisé chez le poisson après la séparation entre mammifères et téléostes, certainement par duplication génique locale. BALM a une expression similaire à celle de BAFF et APRIL chez le poisson, suggérant fortement une fonction dans l'immunité humorale pour le produit de ce gène. Ainsi les mécanis- mes de contrôle de l'immunité humorale seraient plus sophistiqués chez les poissons que chez les mammifères supérieurs, tout du moins au niveau de la sous-famille dont APRIL fait partie.

\section{L'implication d'APRIL}

dans le développement tumoral

L'ARNm d'APRIL a été initialement détecté dans diverses tumeurs solides $[2,3]$, et une fonction promotrice de tumeurs solides a d'abord été attribuée à cette protéine [3, 4]. L'identification des récepteurs pour APRIL, BCMA (B-cell maturation antigen ou TNFRSF-17) et $\mathrm{TACl}$ (transmembrane activator, calcium modulator, and cyclophilin ligand interactor ou TNFRSF-13b) a quelque peu changé la donne. Ces récepteurs sont exclusivement exprimés par les cellules hématopoïétiques, exclusivement sur les lymphocytes B pour BCMA, sur les lymphocytes $B$ mais aussi $T$ et macrophages pour $\mathrm{TACl}$. II est vraisemblable que la fonction promotrice de tumeurs solides soit donc due à l'activité des protéoglycanes à héparane

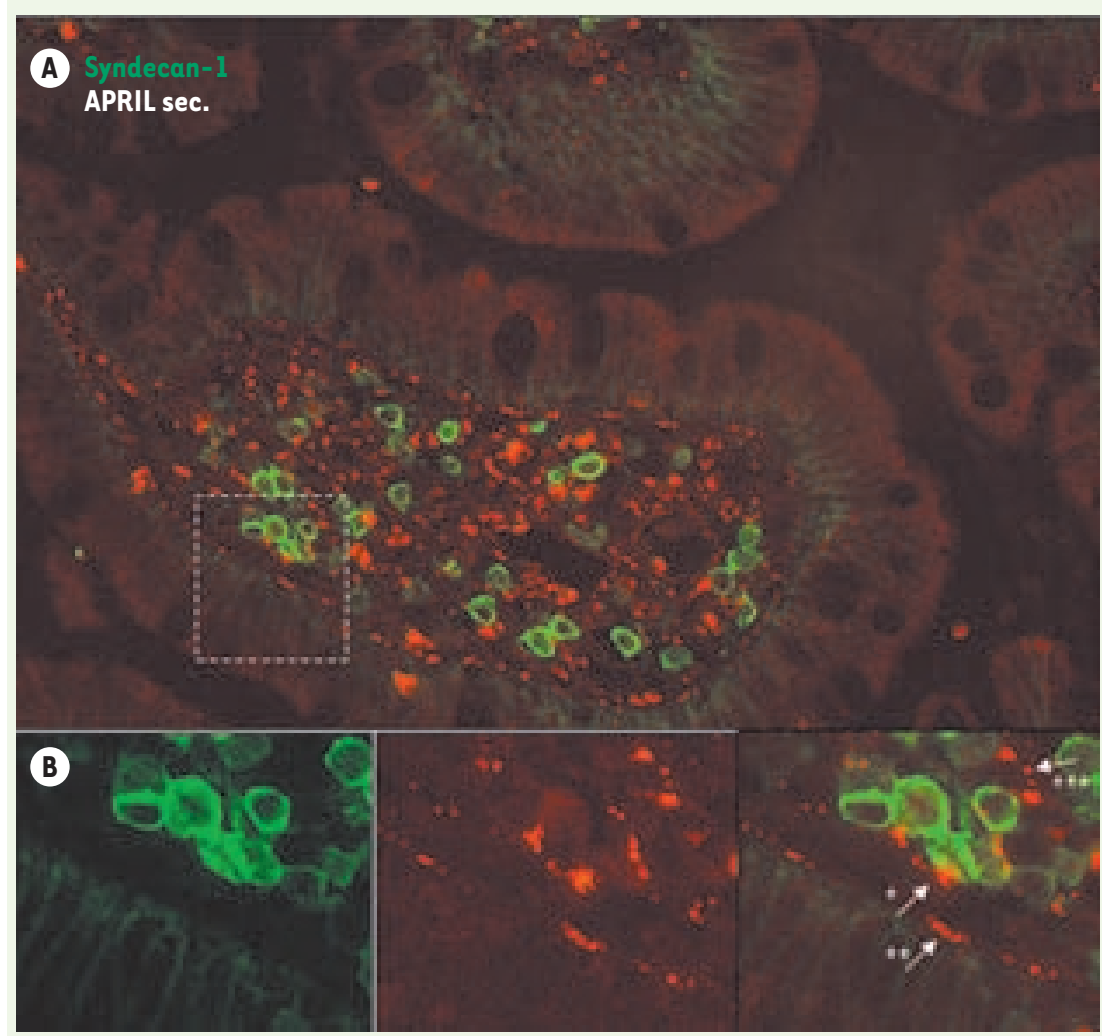

sulfate (HSPG), récemment identifiés comme co-récepteurs d'APRIL $[5,6]$, et qui peuvent faciliter le développement de certaines tumeurs. Néanmoins, même si les interactions APRIL/HSPG devront faire l'objet de travaux supplémentaires clarifiant leur implication dans la tumorigenèse, on peut d'ores et déjà affirmer que ce rôle promoteur est relativement faible. $\varepsilon$ n effet, bien que les interactions APRIL/HSPG puissent promouvoir le développement naturel d'une tumeur solide in vivo dans un modèle animal ou bien in vitro dans une culture cellulaire, l'effet de ce signal promoteur disparait sous l'effet cytotoxique de la chimiothérapie chez les patients porteurs de tumeurs solides [7]. II n'en est pas de même pour les tumeurs d'origine lymphocytaire $B$. En effet, l'effet promoteur d'APRIL a été récemment observé pour ces dernières non seulement dans des modèles précliniques, mais aussi chez les patients sous traitements chimiothérapeutiques. Dans ce cas, une surexpression d'APRIL, détectable dans le sérum et dans les lésions tumorales, est associée avec une plus courte durée de vie pour des patients atteints de leucémie lymphoïde chronique [8] et de lymphome B diffus à grandes cellules [9] respectivement. Cela s'explique par le puissant signal de survie donné par APRIL dans les cellules tumorales de type $B$ grâce à l'expression des récepteurs $\mathrm{TACl}$ et/ou BCMA, conférant à ces dernières une résistance contre des drogues chimiothérapeutiques [10].

Figure 2. Des niches riches en APRIL dans les muqueuses. A. Un double marquage syndécan-1 (vert) et APRIL sécrétée (rouge) dans les villi de la lamina propria montre la colocalisation d'APRIL sécrétée et de plasmocytes exprimant le syndécan-1. B. APRIL se fixe directement sur les plasmocytes exprimant le syndécan-l $(*)$, sur l'épithélium basal exprimant le syndécan-1 $(\star *)$, ou sur des cellules sous-épithéliales n'exprimant pas le syndécan-l ( $\star \star \star)$. 


\section{Des niches riches en APRIL} pour les plasmocytes

Bien que très proches et associées dans une même sous-famille, il serait erroné de penser que BAFF et d'APRIL n'ont que des fonctions complètement redondantes. L'établissement de souris génétiquement déficientes pour l'une ou l'autre de ces molécules a clairement montré le rôle primordial de BAFF dans l'établissement d'un répertoire $B$ mature, alors qu'APRIL a un rôle plus discret, focalisé dans la régulation de la production d'anticorps [11]. Ces divergences de fonction correspondent très vraisemblablement à une différence de récepteurs, puisque BAFF se lie à un récepteur unique BAFF- $R$ (TNFRSF$13 \mathrm{c}$ ), alors qu'APRIL peut interagir, outre les récepteurs $\mathrm{BCMA}$ et $\mathrm{TACl}$, avec les HSPG. En plus des données issues de l'analyse des souris génétiquement déficientes, des expériences in vitro ont montré qu'APRIL avait un rôle dans la survie des cellules $B$ proliférant après stimulation antigénique, pouvait parti- ciper à l'induction de la commutation isotypique T-dépendante associée au ligand de CD40 (TNFSF-5), mais surtout était un puissant inducteur de la commutation isotypique T-indépendante. Cette dernière fonction dépend de $\mathrm{TACl}$, bien que le rôle de ce récepteur semble subtil, car une fonction opposée, inhibitrice de l'activation du lymphocyte B lui a aussi été attribuée. Dans ces dernières expériences, BAFF semble partager le rôle d'APRIL.

L'étude de l'expression in situ d'APRIL a apporté récemment des indications précises sur le rôle in vivo de cette molécule chez l'homme. En effet, l'utilisation d'un anticorps reconnaissant sélectivement le produit sécrété d'APRIL a révélé de fortes concentrations de cette molécule au niveau de la zone sous-épithéliale des organes lymphoïdes associés aux muqueuses, comme l'amygdale et l'intestin grêle [12] (Figure 2). Les concentrations en APRIL sont si importantes et distribuées de façon si précise que l'on peut

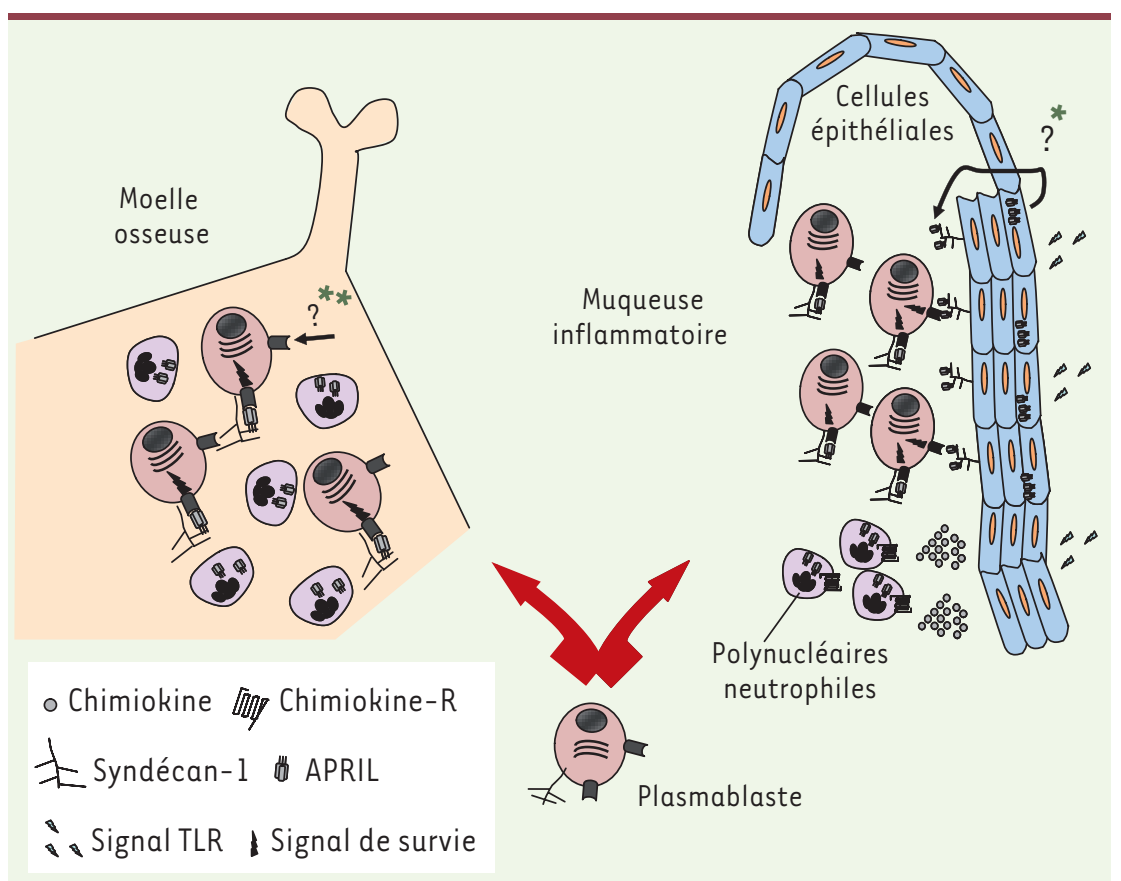

Figure 3. APRIL fait le printemps des plasmocytes. Après sa naissance dans un centre germinatif à partir d'un lymphocyte B, le plasmablaste entre dans la circulation sanguine et se dirige soit dans la moelle osseuse pour y persister et assurer une protection systémique, soit dans les muqueuses siège d'un processus inflammatoire pour assurer une protection locale. Dans la moelle osseuse, la survie des plasmocytes est assurée par APRIL produite de manière constitutive par les granulocytes. Dans les muqueuses et dans un contexte inflammatoire, la stimulation des récepteurs Toll (TLR) exprimés par les cellules épithéliales apicales conduit à la production de chimiokines attirant les polynucléaires neutrophiles producteurs d'APRIL, mais aussi à la synthèse d'APRIL par ces mêmes cellules épithéliales. APRIL sécrétée est retenue localement par le syndécan-l exprimé au niveau de l'épithélium basal. *Le mécanisme permettant le transport

dirigé d'APRIL sécrétée de la zone apicale vers la zone basale reste à élucider. Ainsi, les plasmocytes trouvent un milieu favorable à leur survie dans la zone sous-épithéliale des muqueuses. Les plasmocytes répondent au signal de survie d’APRIL grâce à leur expression de BCMA et syndécan-1 pour un signal en cis, ou de BCMA pour un signal en trans. ** Les zones de retention secrété dans la moelle osseuse n’ont pas encore été rapportées, si bien que l'on ne sait pas si un signal de survie en trans existe dans cet organe. 
syndécan-1, ou bien également à des cellules encore mal définies présentes dans les villosités de la lamina propria, mais toujours proches des plasmocytes. Dans le premier cas, le signal de survie d'APRIL pour les plasmocytes est donné en cis, alors que dans les deux derniers cas il est donné en trans. Un second anticorps a permis de détecter sélectivement les cellules produisant APRIL in situ. Dans les muqueuses, la production d'APRIL est assurée principalement par les polynucléaires neutrophiles et à un degré moindre par les cellules épithéliales de l'amygdale. La Figure 2 montre la production d'APRIL ainsi que la rétention d'APRIL sécrétée dans les villosités de la lamina propria associée à l'intestin grêle. La présence des neutrophiles dans ces organes ainsi que la production d'APRIL par l'épithélium requièrent la présence d'un pathogène. Un rôle d'APRIL pour la survie des plasmocytes a été aussi établi récemment au niveau de la moelle osseuse [14]. Dans ce tissu, la production d'APRIL est constitutive, assurée par les précurseurs des polynucléaires en voie de maturation ou déjà matures. II n'est pas sûr que BAFF partage avec APRIL le rôle de survie pour les plasmocytes qu'on lui a attribué [15]. En effet, la survie des plasmocytes dans la moelle osseuse n'est pas affectée par l'absence de BAFF [14]. De plus, les plasmocytes n'expriment ni BAFF- $R$ ni $\mathrm{TACl}$, mais seulement $B C M A$, qui possède une affinité nettement supérieure pour APRIL que pour BAFF [16].
Au total, ces études récentes permettent de mieux définir le rôle d'APRIL dans la biologie des plasmocytes et le contrôle de la réponse humorale en général. La Figure 3 résume le rôle d'APRIL sur les plasmocytes de la moelle osseuse et des muqueuses. Ces résultats expliquent, au moins en partie, pourquoi la moelle osseuse est un tissu si approprié pour les plasmocytes: la production constitutive d'APRIL dans ce tissu permet leur survie prolongée pendant des années. En revanche, l'environnement des muqueuses ne peut assurer aux plasmocytes qu'une survie de courte durée, correspondant à la durée de vie des niches riches en APRIL ainsi qu'à la présence d'une réaction inflammatoire. $\diamond$

\section{APRIL, the spring of plasmocytes}

\section{REMERCIEMENTS}

Le soutien financier du Fond National pour la Recherche Suisse, et des fondations Leenards et Jacques et Floria Gossweiler et Dr Henri Dubois-Ferrière/Dinu Lipatti est vivement remercié.

\section{RéFÉRENCES}

1. Glenney GW, Wiens GD. Early diversification of the TNF superfamily in teleosts: genomic characterization and expression analysis. J Immunol 2007 ; 178 : 7955-73.

2. Kelly K, Manos $\varepsilon$, Jensen G, et al. APRIL/TRDL-1, a tumor necrosis factor-like ligand, stimulates cell death. Cancer Res $2000 ; 60$ : 1021-7.

3. Hahne M, Kataoka T, Schroter M, et al. APRIL, a new ligand of the tumor necrosis factor family, stimulates tumor cell growth.J Exp Med 1998; 188 : 1185-90.

4. Rennert $P$, Schneider $P$, Cachero TG, et al. A soluble form of $B$ cell maturation antigen, a receptor for the tumor necrosis factor family member APRIL, inhibits tumor cell growth. J Exp Med 2000 ; 192 : 1677-84.
5. Ingold K, Zumsteg A, Tardivel A, et al. Identification of proteoglycans as the APRIL-specific binding partners. J Exp Med $2005 ; 201: 1375-83$.

6. Hendriks J, Planelles L, de Jong-Odding J, et al. Heparan sulfate proteoglycan binding promotes APRIL-induced tumor cell proliferation. Cell Death Differ $2005 ; 12: 637-48$.

7. Mhawech-Fauceglia P, Allal AK, Odunsi K, et al. Role of APRIL in the development of solid tumors: retrospective studies in human carcinomas. Eur J Cancer 2008 (sous presse).

8. Planelles L, Castillo-Gutierrez S, Medema JP, et al. APRIL but not BLyS serum levels are increased in chronic lymphocytic leukemia: prognostic relevance of APRIL for survival. Haematologica 2007 ; 92: 1284-5.

9. Schwaller J, Schneider P, Mhawech-Fauceglia P, et al. Neutrophil-derived APRIL concentrated in tumor lesions by proteoglycans correlates with human B-cell lymphoma aggressiveness. Blood 2007 ; 109 : 331-8.

10. He B, Chadburn A, Jou $\varepsilon$, et al. Lymphoma B cells evade apoptosis through the TNF family members BAFF/BLyS and APRIL. J Immunol. 2004 ; $172: 3268-3279$.

11. Castigli $\varepsilon$, Scott S, Dedeoglu F, et al. Impaired IgA class switching in APRIL-deficient mice. Proc Natl Acad Sci USA 2004 ; 101 : 3903-8.

12. Huard B, McKee T, Bosshard C, et al. APRIL secreted by neutrophils binds to heparan sulfate proteoglycans to create plasma cell niches in human mucosa. J Clin Invest 2008; $118: 2887-95$

13. Brandtzaeg P, Johansen FE. Mucosal B cells: phenotypic characteristics, transcriptional regulation, and homing properties. Immunol Rev 2005; 206: 32-63.

14. Belnoue $\varepsilon$, Pihlgren M, McGaha TL, et al. APRIL is critical for plasmablast survival in the bone marrow and poorly expressed by early life bone marrow stromal cells. Blood 2008 (sous presse).

15. O'Connor BP, Raman VS, Erickson LD, et al. BCMA is essential for the survival of long-lived bone marrow plasma cells. J Exp Med 2004 ; 199 : 91-8.

16. Day $\varepsilon S$, Cachero TG, Pian F, et al. Selectivity of BAFF/ BLyS and APRIL for binding to the TNF family receptors BAFFR/BR3 and BCMA. Biochemistry 2005 ; $44: 1919-31$

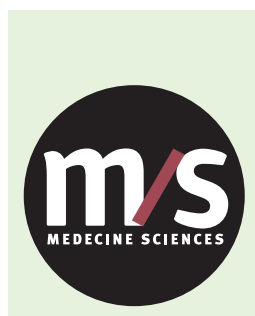

$>$ Grâce à $m / s$, vous vivez en direct

Tarifs d'abonnement M/S - 2009

les progrès des sciences biologiques et médicales

Abonnez-vous

à Médecine/Sciences

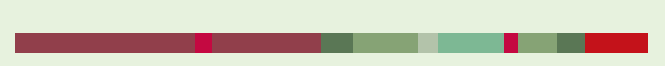
Bulletin d'abonnement page 30 dans ce numéro de $\mathrm{m} / \mathrm{s}$
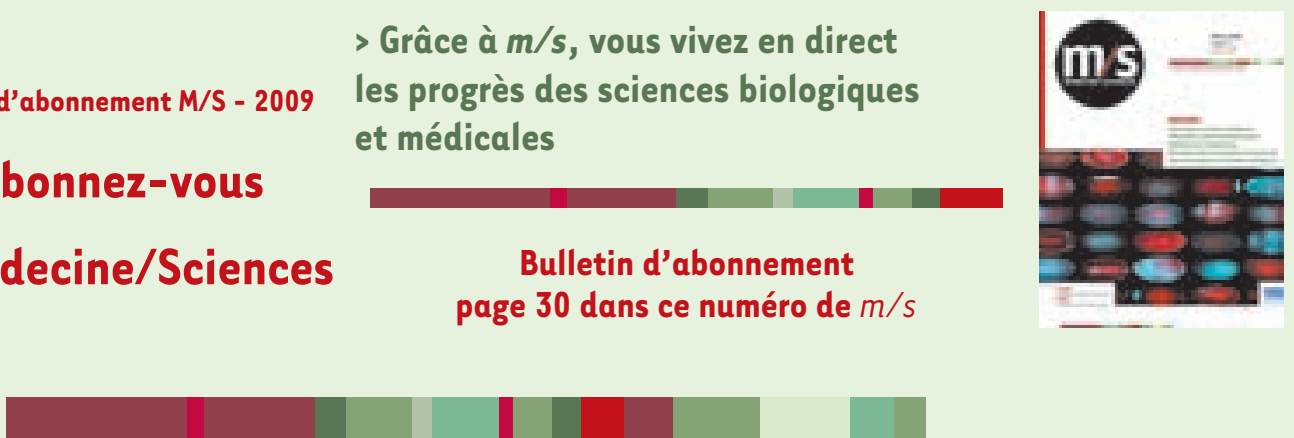